University of Nebraska - Lincoln

DigitalCommons@University of Nebraska - Lincoln

\title{
Novel Small Molecule Inhibitors of Botulinum Neurotoxin a Metalloprotease Activity
}

James C. Burnett

Developmental Therapeutics Program, NCI Frederick, Frederick, MD

James J. Schmidt

US Army Medical Research Institute of Infectious Diseases, Frederick, MD 21702, USA

Robert G. Stafford

US Army Medical Research Institute of Infectious Diseases, Frederick, MD 21702, USA

Rekha G. Panchal

Developmental Therapeutics Program, NCI Frederick, Frederick, MD

Tam L. Nguyen

Developmental Therapeutics Program, NCI Frederick, Frederick, MD

See next page for additional authors

Follow this and additional works at: https://digitalcommons.unl.edu/usarmyresearch

Part of the Operations Research, Systems Engineering and Industrial Engineering Commons

Burnett, James C.; Schmidt, James J.; Stafford, Robert G.; Panchal, Rekha G.; Nguyen, Tam L.; Hermone, Ann R.; Vennerstrom, Jonathan L.; McGrath, Connor F.; Lane, Douglas J.; Sausville, Edward A.; Zaharevitz, Daniel W.; Gussio, Rick; and Bavari, Sina, "Novel Small Molecule Inhibitors of Botulinum Neurotoxin a Metalloprotease Activity" (2003). US Army Research. 20.

https://digitalcommons.unl.edu/usarmyresearch/20

This Article is brought to you for free and open access by the U.S. Department of Defense at DigitalCommons@University of Nebraska - Lincoln. It has been accepted for inclusion in US Army Research by an authorized administrator of DigitalCommons@University of Nebraska - Lincoln. 


\section{Authors}

James C. Burnett, James J. Schmidt, Robert G. Stafford, Rekha G. Panchal, Tam L. Nguyen, Ann R.

Hermone, Jonathan L. Vennerstrom, Connor F. McGrath, Douglas J. Lane, Edward A. Sausville, Daniel W.

Zaharevitz, Rick Gussio, and Sina Bavari 


\title{
Novel small molecule inhibitors of botulinum neurotoxin A metalloprotease activity
}

\author{
James C. Burnett, ${ }^{\mathrm{a}}$ James J. Schmidt, ${ }^{\mathrm{b}}$ Robert G. Stafford, ${ }^{\mathrm{b}}$ Rekha G. Panchal, ${ }^{\mathrm{a}}$ \\ Tam L. Nguyen, ${ }^{\mathrm{a}}$ Ann R. Hermone, ${ }^{\mathrm{a}}$ Jonathan L. Vennerstrom, ${ }^{\mathrm{c}}$ Connor F. McGrath, ${ }^{\mathrm{a}}$ \\ Douglas J. Lane, ${ }^{\mathrm{a}}$ Edward A. Sausville, ${ }^{\mathrm{a}}$ Daniel W. Zaharevitz, ${ }^{\mathrm{a}}$ Rick Gussio, ${ }^{\mathrm{a}, *}$ \\ and Sina Bavari ${ }^{\mathrm{b}, *}$ \\ a Developmental Therapeutics Program, NCI Frederick, Frederick, MD 21702, USA \\ ${ }^{\mathrm{b}}$ US Army Medical Research Institute of Infectious Diseases, Frederick, MD 21702, USA \\ ${ }^{c}$ College of Pharmacy, University of Nebraska Medical Center, 986025 Nebraska Medical Center, Omaha, NE 68198-6025, USA
}

Received 15 August 2003

\begin{abstract}
Botulinum neurotoxins (BoNTs) are among the most lethal biological substances to have been weaponized and are listed as biodefense category A agents. Currently, no small molecule (non-peptidic) therapeutics exist to counter this threat; hence, identifying and developing compounds that inhibit BoNTs is a high priority. In the present study, a high-throughput assay was used to identify small molecules that inhibit the metalloprotease activity of BoNT serotype A light chain (BoNT/A LC). All inhibitors were further verified using a HPLC-based assay. Conformational analyses of these compounds, in conjunction with molecular docking studies, were used to predict structural features that contribute to inhibitor binding and potency. Based on these results, a common pharmacophore for BoNT/A LC inhibitors is proposed. This is the first study to report small molecules (non-peptidics) that inhibit BoNT/A LC metalloprotease activity in the low $\mu \mathrm{M}$ range.
\end{abstract}

(C) 2003 Elsevier Inc. All rights reserved.

Keywords: Bioterrorism; Botulinum neurotoxin; Drug discovery; High-throughput screen; Inhibitors; Molecular modeling; Pharmacophore; Threedimensional database search; Metalloprotease

Botulinum neurotoxins (BoNTs) are produced by spore forming anaerobic bacteria Clostridium botulinum and are among the most lethal of biological poisons $[1,2]$. Seven immunologically distinct BoNT serotypes (designated A-G) have been identified [3]. Accidental exposure to BoNTs, for example through contaminated food, can result in life threatening flaccid paralysis [4]. Furthermore, BoNTs have been weaponized in highly toxic aerosol form, and consequently pose a significant "dual threat"-i.e., both to civilian and military populations $[5,6]$. As a result, there is an urgent need for therapeutic countermeasures against BoNTs [7].

\footnotetext{
${ }^{*}$ Corresponding authors. Fax: 1-301-846-6106 (R. Gussio), Fax: 1-301-619-2348 (S. Bavari).

E-mail addresses: Gussio@NCIFCRF.GOV (R. Gussio), Sina. Bavari@AMEDD.ARMY.MIL (S. Bavari).
}

BoNT is secreted as a holotoxin composed of two peptide chains that are linked by a disulfide bridge [8]. The heavy chain is responsible for: (1) targeting and binding to surface receptors on nerve terminals; (2) translocation into the neuronal cytosol via the formation of a low $\mathrm{pH}$ endosome; and (3) protecting the substrate binding cleft of the light chain prior to neuronal internalization $[9,10]$. The light chain, which dissociates from the heavy chain in the low endosomal $\mathrm{pH}$, is released into the cytosol where it acts as a zinc metalloprotease that cleaves SNARE (soluble NSF-attachment protein receptor) proteins: SNAP-25 (synaptosomal-associated protein of $25 \mathrm{kDa}$ ), synaptobrevin, and syntaxin $[9,10]$. BoNT serotypes A, C, and E cleave SNAP-25 [11]; serotypes B, D, F, and G cleave synaptobrevin [12-15]; and serotype $\mathrm{C}$ can also use syntaxin as substrate [16]. Without functional 
SNARE complexes, acetylcholine is not released into neuromuscular junctions, leading to paralysis.

Research to identify peptide and small molecule inhibitors of BoNT serotype A (BoNT/A) has targeted both holotoxin translocation and light chain (BoNT/A LC) metalloprotease activity. Sheridan et al. [17] and Deshpande et al. [18] have shown that a number of antimalarial agents interfere with BoNT/A translocation into nerve cytoplasm. Specifically, it has been shown that several antimalarial compounds act subsequent to toxin binding to cell-surface receptors [17], and it has been hypothesized that these agents inhibit BoNT/A cytosol entry by raising endosomal $\mathrm{pH}$ (an endosomal $\mathrm{pH}$ of 5.5 or lower is needed for release into the cytoplasm) [17,18]. Hayden et al. [19] have found that BoNT/A LC is inhibited by $\mathrm{mM}$ concentrations of known protease inhibitors: captopril, lysinopril, and enalapril. In the same study, it was also reported that a number of short peptides, from specific "hinge" libraries, inhibit BoNT/A LC activity by as much as $51 \%$ at concentrations as low as $0.5 \mu \mathrm{M}$ [19]. Using a chromatographic method, Schmidt et al. [20] identified the peptide motif CRATKML as a potent inhibitor. In a subsequent study, the Cys residue of CRATKML was replaced with thiol containing organic moieties and it was found that a 2-mercapto3-phenylpropionyl containing derivative was the most effective $\left(K_{\mathrm{i}}=0.3 \mu \mathrm{M}\right)[21]$.

Presently, no small molecule (non-peptidic) inhibitors of BoNT/A LC metalloprotease activity, which are effective in the low $\mu \mathrm{M}$ range, have been reported. In this report, a high-throughput assay was used to facilitate BoNT/A LC inhibitor identification [22]. Initially, the National Cancer Institute (NCI) Diversity set was screened, and several compounds possessing $>50 \%$ inhibition (at $20 \mu \mathrm{M}$ concentration) were identified. Based on molecular modeling studies, common structural features and binding modes in the BoNT/A LC substrate binding cleft were identified for these leads. Additional screening of 7-chloro-4-aminoquinoline derivatives also resulted in the identification of leads that substantially inhibit BoNT/A LC at $20 \mu \mathrm{M}$ concentrations. Analyses of these compounds indicate that they share common structural/functional group characteristics with inhibitors identified during the screen of the NCI Diversity set, and that they may also bind to the enzyme's substrate binding cleft in a similar manner. Based on our analyses, a common pharmacophore for BoNT/A LC inhibitors is proposed.

\section{Materials and methods}

Compounds. The NCI Diversity set was obtained in 96-well plate format from the National Cancer Institute (http://dtp.nci.nih.gov/ branches/dscb/diversity_explanation.html). The syntheses of $N$, $N$-bis(7-chloroquinolin-4-yl)alkanediamines and $N, N$-bis(7-chloroquinolin-4-yl)heteroalkanediamines used in these studies have been described previously [23,24]. Amodiaquine, chloroquine, quinacrine, quinidine, and quinine were obtained from Sigma-Aldrich (USA).

High-throughput assay of BoNT/A LC activity. The high-throughput assay used to screen BoNT/A LC proteolytic activity has been described previously [22]. Briefly, liquid transfers were done with a SciClone automated pipettor (Zymark, Hopkinton, MA). Recombinant BoNT/A LC [25] was diluted to $0.6 \mu \mathrm{g} / \mathrm{ml}$ in $40 \mathrm{mM}$ Hepes, and $0.5 \mathrm{mg} / \mathrm{ml} \mathrm{BSA}, \mathrm{pH} 7.4$. Test compounds was diluted to $0.2 \mathrm{mM}$ with methyl sulfoxide. Ten microliters of each compound were transferred into the corresponding wells of another 96-well plate containing immobilized fluorescent-labeled peptide substrate, specific for the protease activity of BoNT/A LC [22]. Ninety microliters BoNT/A LC was then added and mixed, for final concentrations of $20 \mu \mathrm{M}$ compound and $0.5 \mu \mathrm{g} / \mathrm{ml}$ BoNT/A LC. Blanks (buffer only) and controls (buffer and BoNT/A LC), eight wells each, were included on each assay plate. After incubation at $30^{\circ} \mathrm{C}$ for $60 \mathrm{~min}, 70 \mu \mathrm{l}$ was transferred from each well to an opaque-wall plate and fluorescence was measured in a Wallac 1420 multi-label counter (Perkin-Elmer, Gaithersburg, MD).

HPLC-based assay of BoNT/A LC protease activity. Compounds exhibiting $\geqslant 40 \%$ inhibition in high-throughput assays were retested in a HPLC-based assay as described previously $[1,26]$.

BoNT/A LC refinement and inhibitor docking. The structure of BoNT/A LC was obtained from the deposited crystallographic coordinates of the holotoxin (PDB refcode = 3BTA) [27]. The $3.2 \AA$ holotoxin includes the heavy chain, light chain, and a protective residue belt that covers the substrate binding site of the light chain. The heavy chain and the protective belt were removed from the holotoxin, and the light chain was energy refined using the Discover (Accelrys, San Diego, CA) program's cff91 force field. During the refinement, the zinc ion and residues coordinating the zinc ion were fixed in their original coordinates. Our strategy entailed applying $2000 \mathrm{kcal} / \mathrm{mol}$ per $\AA^{2}$ of force that was stepped off the structure in $100-\mathrm{kcal} / \mathrm{mol}$ decrements by minimizing with conjugate gradients, until the norm of the gradient was $0.01 \mathrm{kcal} / \AA$ [28]. This process was repeated until all applied external force was removed. The resulting coordinates of the final model were within the experimentally determined X-ray crystallographic resolution. The optimized structure had an rms deviation of $2.6 \AA$ across all backbone atoms from the reported crystallographic coordinates of the holotoxin light chain.

Using InsightII molecular modeling software (Accelrys, San Diego, CA), identified inhibitors were manually docked into the BoNT/A LC substrate binding cleft, and van der Waals violations of $0.25 \AA$ were removed by small adjustments to side-chain torsion angles and inhibitor positioning. The inhibitor-enzyme structure coordinates were subsequently tether minimized in the same manner as described above and the final structure was subjected to hydropathic analysis using the program HINT (eduSoft, Richmond, VA).

Conformer generation. Conformational models of inhibitors were generated using Catalyst 4.7 software (Accelrys, San Diego, CA). Inhibitors were imported into Catalyst and energy minimized to the closest local minimum using the generalized CHARMM-like force field, as implemented in the program. Following, the "best quality" conformational search option was employed to generate conformers within $20 \mathrm{kcal} / \mathrm{mol}$ from the global energy minimum. In this study, it was found that 100 conformers per compound ensured adequate coverage of conformational space.

\section{Results and discussion}

\section{Screening of the NCI diversity set}

A high-throughput fluorescence-based assay was initially used to screen the NCI diversity set: a collection of 1990 molecules that were selected to cover a wide range 
of conformational space, and at the same time provide pharmacophore diversity and structural rigidity. Following the initial high-throughput screen, a HPLCbased assay $[1,26]$ was used to eliminate false positives resulting from fluorescence quenching by some of the compounds. The final set of inhibitors, which were tested at $20 \mu \mathrm{M}$ concentration in the presence $0.1 \mathrm{mM}$ substrate, consisted of 21 compounds with potencies ranging from $14 \%$ to $100 \%$ BoNT/A LC inhibition. Two-dimensional structures for compounds possessing $>40 \%$ inhibition are shown in Table 1 .
Additional testing of NSC 625324 (silver sulfadiazine), initially the most potent of the identified compounds, indicated that this molecule's efficacy was entirely mediated by the silver ion: no inhibition was observed when the unionized, organic form of sulfadiazine was tested in the HPLC-based assay. Furthermore, BoNT/A LC activity was examined in the presence of silver acetate, and $100 \%$ inhibition of protease activity was found at concentrations $\geqslant 5 \mu \mathrm{M}$ silver ion (the $\mathrm{IC}_{50}$ was $1.5-2.0 \mu \mathrm{M})$. Cesium and rubidium salts did not inhibit BoNT/A LC protease activity at concentrations

Table 1

NCI diversity set inhibitors of BoNT/A LC

\begin{tabular}{ll}
\hline Compound & NSC No. \\
\hline & NSC 625324 (silver sulfadiazine)
\end{tabular}

$=\stackrel{\mathrm{I}}{\mathrm{O}} \underset{\mathrm{O}}{\ominus} \underset{\mathrm{Ng}}{\ominus} \overbrace{\mathrm{N}}^{\mathrm{N}}=$

NSC 625324 (silver sulfadiazine)

100

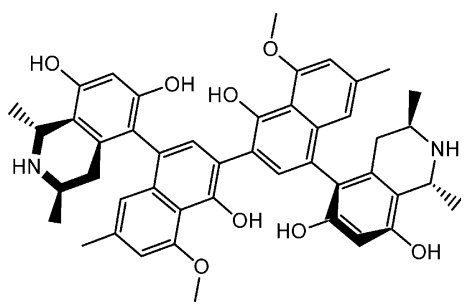

NSC 661755 (michellamine B)

62

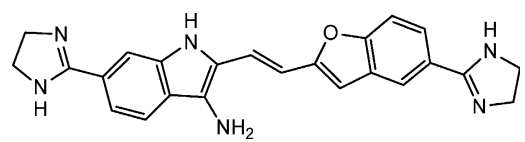

NSC 357756<smiles></smiles><smiles>CCOc1ccc2c(ccc(/C=C/c3ccc4c(C)ccnc4c3O)[n+]2C)c1</smiles><smiles>O=[N+]([O-])c1cc(Cc2cc([N+](=O)[O-])c3cccnc3c2O)c(O)c2ncccc12</smiles><smiles>[O-]c1c2ccc(Nc3ccc4c(c3)nc3ccc(I)cc3[n+]4-c3ccccc3)cc2nc2ccc(I)cc12</smiles>

\footnotetext{
${ }^{a}$ Compounds tested in the HPLC-based assay at $20 \mu \mathrm{M}$ concentration in the presence of $0.1 \mathrm{mM}$ substrate.
} 
up to $20 \mu \mathrm{M}$. The presence of zinc $(5-50 \mu \mathrm{M})$ in assays had no effect on inhibition by $3 \mu \mathrm{M}$ silver ion, but addition of $1 \mathrm{mM}$ DTT immediately reversed inhibition. These observations suggest that silver neither displaces zinc from the active site, nor causes irreversible denaturation by reacting with a residue important for conformational stability. It is possible that this ion might bind to polar residues in or near the substrate binding cleft, blocking access to the catalytic site.

Inhibitors NSC 86372 and 130796 both contain an 8hydroxyquinoline moiety (Table 1), which is known to chelate metal ions [29]. Based on our analyses, both of these molecules do chelate the BoNT/A LC catalytic zinc. Support for a chelation mechanism is based on: (1) assay data showing that several congeners of NSC 86372 possess equivalent inhibitory potency to that of the original lead molecule; (2) addition of $20 \mu \mathrm{M}$ zinc immediately and quantitatively reversed inhibition caused by up to $10 \mu \mathrm{M}$ NSC 86372 ; and (3) NSC 86372, its congeners, and NSC 130796 inhibit other zinc metalloproteases (including anthrax lethal factor and BoNT serotype B light chain (BoNT/B LC)). Consequently, the 8-hydroxyquinoline motif is not suitable for further development as a component of BoNT/A LC inhibitors; however, as will be shown, structural similarities between NSC 86372 and other identified compounds do appear to fit a general model for BoNT/A LC inhibition.

\section{Screening of 7-chloro-4-aminoquinoline derivatives}

Congeneric series of $N, N$-bis(7-chloroquinolin-4yl)alkanediamines [23] and $N, N$-bis(7-chloroquinolin-4yl)heteroalkanediamines [24] (collectively referred to as BQs for the remainder of the text) were examined for BoNT/A LC inhibition. The compounds were tested in the HPLC-based assay at $20 \mu \mathrm{M}$ concentration in the presence of $0.1 \mathrm{mM}$ substrate $[1,26]$. Inhibition of BoNT/A LC by these compounds was not affected by added zinc. Furthermore, when tested against BoNT/B LC protease activity, no significant inhibition was found. Taken together, these observations suggest that these compounds exhibit specificity for binding to BoNT/A LC and are not zinc chelators. Table 2 shows the two-dimensional structures of these compounds, along with percent inhibition of BoNT/A LC.

Results from our studies on BQs led us to hypothesize that other quinoline-based compounds might also inhibit BoNT/A LC enzymatic activity. One method for fast tracking the evolution of therapeutics against BoNT/A LC would be to identify inhibitors of this enzyme that are already approved drugs (and subsequently already possess pharmacological and toxicological data from clinical trials). Subsequently, we tested five readily available antimalarial drugs-amodiaquine, chloroquine, quinacrine, quinidine, and quinine-that share similar structural characteristics with the BQs. These compounds were obtained and examined in our HPLCbased assay; structures of these compounds and percent inhibition of BoNT/A LC protease activity are shown in Table 2.

\section{Identification of structural similarities and potential binding sites for inhibitors from the NCI diversity set}

Superimpositions of several of the most potent inhibitors identified during the NCI Diversity set screen are shown in Fig. 1. An evident feature to emerge from superimposing these compounds is that they all possess biaryl and triaryl heterocyclic scaffolds (for example, quinoline, phenazinium, or xanthene). The exception to this observation is michellamine B-the central naphthalenes of this molecule do not possess heteroatoms. However, like aromatic heterocyclic nitrogens or oxygens, hydroxy and methoxy substituents on the naphthalene rings may participate in electron donating interactions. The superimposed structures in Fig. 1 also indicate that possessing hydrophobic moieties at either end of the aromatic components may be important for potency.

To gain a better understanding of how these inhibitors interact with BoNT/A LC, molecular docking studies were performed. Michellamine B (Table 1) was the first inhibitor to be examined. This molecule was the best lead for initially probing the steric constraints of the substrate binding cleft, as it is an atropisomer that results from hindered rotation of about single bonds [3033]; it possesses restricted conformations, which reduces the number of potential binding modes that it may assume in the enzyme's substrate binding cleft. Furthermore, unlike NSC 86372, inhibition of BoNT/A LC by michellamine $B$ is not affected by added zinc, suggesting that inhibition is not simply the result of michellamine B acting as a chelator.

Fig. 2 shows michellamine B docked in the BoNT/A LC substrate binding cleft. There is a good complement between the steric space of the binding cleft and the twist planar conformation of michellamine B. Hydropathic analyses of favorable and unfavorable contacts in several docked models indicated that the best binding mode results when the naphthalenes (labeled A and B for descriptive purposes, Fig. 2) extend down the length of the catalytic cleft. In this model, the two hydroxyl moieties of the naphthalenes exist in close proximity to the catalytic zinc and form an intramolecular hydrogen bond. Either of the hydroxyl moieties may displace the water molecule that is used by the enzyme's catalytic engine during peptide lysis. As seen in Fig. 2, the methoxy and methyl groups of naphthalene A point toward a hydrophobic pocket (referred to as binding subsite 1 for the remainder of the text) that is formed by the aromatic side-chains of residues Phe 162, Phe 177, and Phe 193, as well as the side-chain methyl of Thr 219. The methoxy 
Table 2

Quinoline-based inhibitors of BoNT/A LC

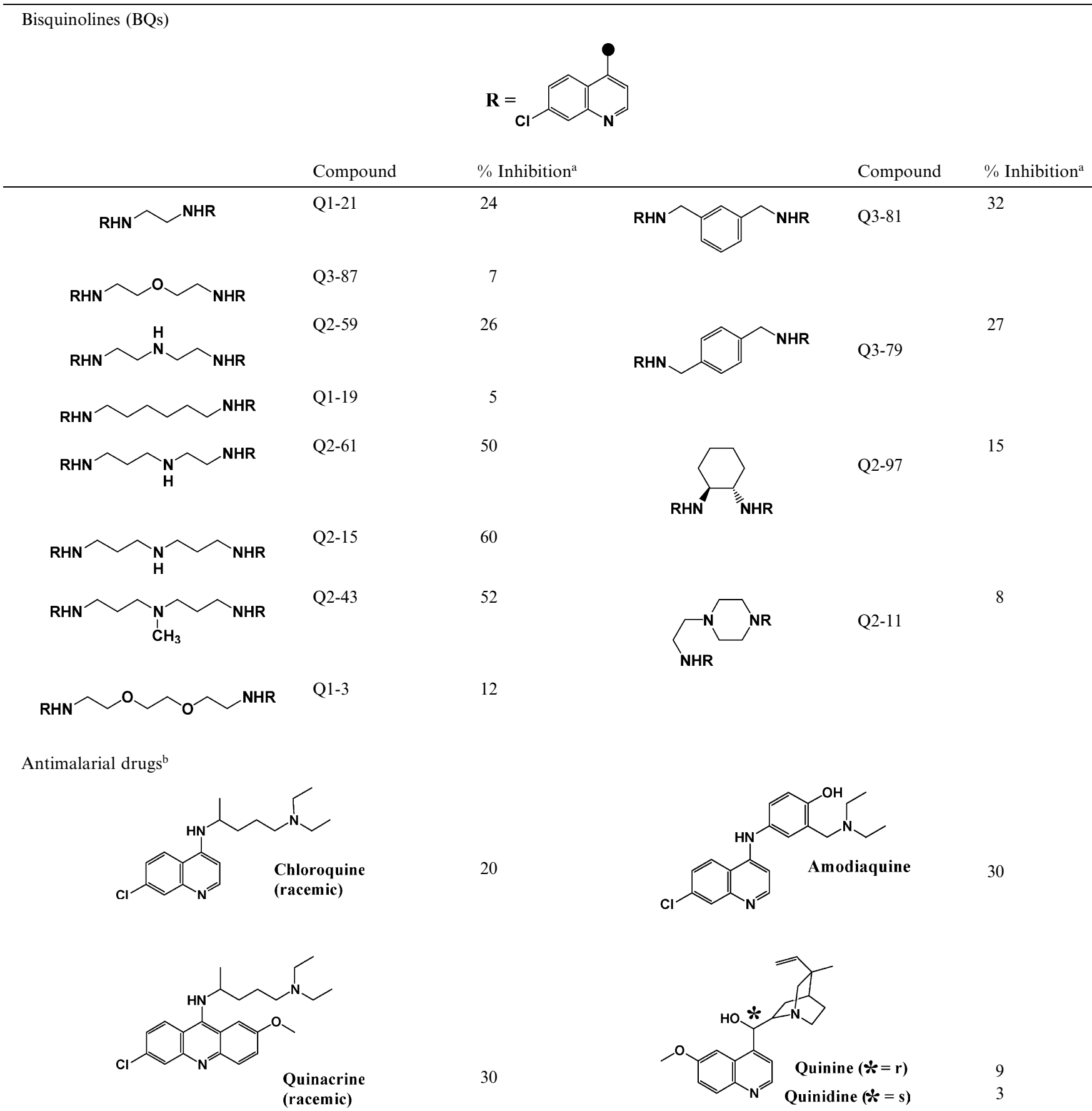

${ }^{\mathrm{a}}$ Compounds tested in the HPLC-based assay at $20 \mu \mathrm{M}$ concentration in the presence of $0.1 \mathrm{mM}$ substrate.

${ }^{\mathrm{b}}$ Antimalarial drugs tested in the HPLC-based assay to $50 \mu \mathrm{M}$ concentration in the presence of $0.2 \mathrm{mM}$ substrate.

moiety of naphthalene B packs into a deep pocket (referred to as binding subsite 2 for the remainder of the text) that is located behind His 226 of the catalytic engine, and surrounded by residues Met 164, Thr 175, Arg 230, and Pro 238. The methyl moiety of naphthalene B packs into space behind the side-chain pyrrolidine of Pro 238.
The tetrahydroisoquinoline attached to naphthalene A binds in such a way that the 6,8-diol moieties point toward the solvent, while the 1,3-dimethyl moieties point toward hydrophobic residues of the substrate binding cleft. The ionizable amine of the tetrahydroisoquinoline attached to naphthalene B points towards a cluster of polar residues, including Glu 55, Gln 161, Glu 


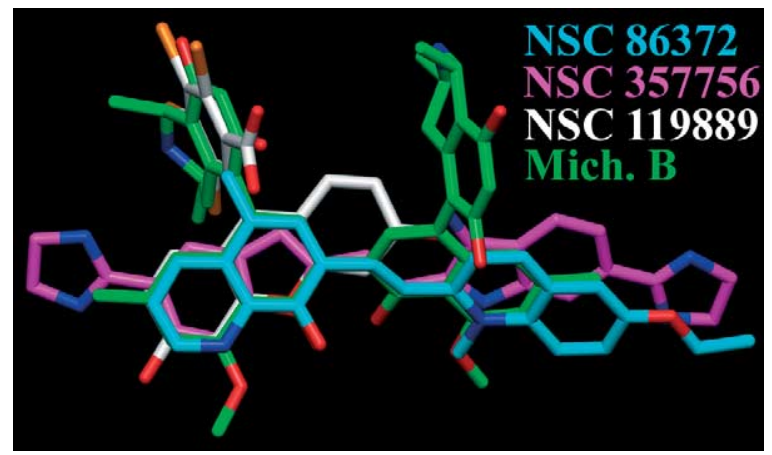

Fig. 1. Overlay of BoNT/A LC inhibitors from the NCI Diversity set. For all structures, nitrogen atoms are blue, oxygen atoms are red, and iodine atoms are orange. Carbon atoms for michellamine B are green, for NSC 86372 are light blue, for NSC 357756 are magenta, and for NSC 119889 are white. There are structural trends in: (1) the superimposed aromatic scaffolds and (2) the positioning of hydrophobic moieties.

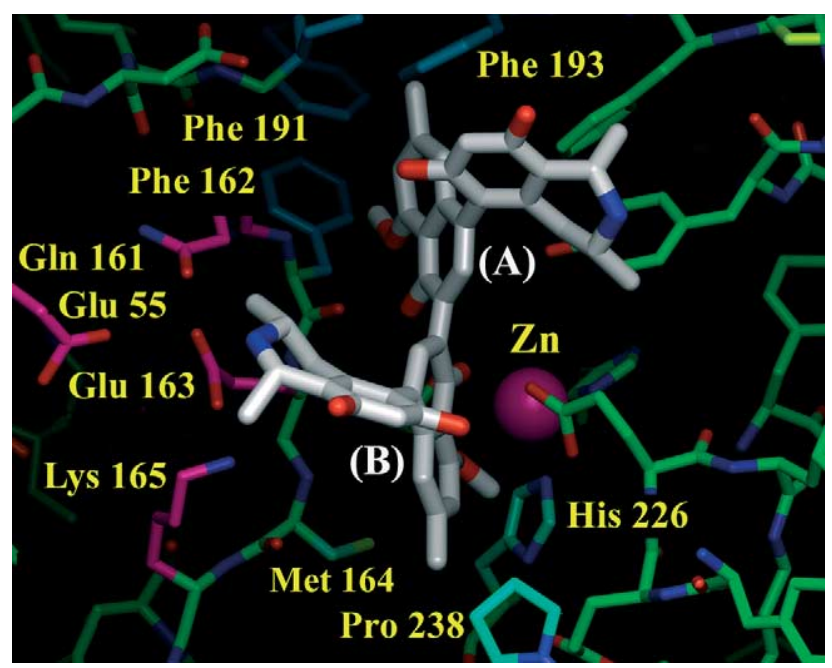

Fig. 2. Michellamine B docked in the BoNT/A LC substrate binding cleft. Nitrogen atoms are blue and oxygen atoms are red. Carbon atoms of michellamine B are white, and the naphthalene scaffolds of the inhibitor are labeled A and B (as referenced in the text). Carbon atoms of residues in predicted binding subsites are light blue (binding subsite 1 in the text), light green (binding subsite 2 in text), and magenta (referred to as the polar region in the text). All other enzyme carbons are green. For michellamine B, the methoxy and methyl substituents of naphthalene A pack into binding subsite 1, while the methoxy of naphthalene B packs into binding subsite 2. The ionizable nitrogen of the tetrahydroisoquinoline attached to naphthalene B points toward a cluster of polar residues: Glu 55, Gln 161, Glu 163, and Lys 165.

163, Lys 165, and Arg 176, and may either engage directly in an ionic bond with one of the Glu residues in this area, or participate in a water mediated hydrogen bond with any of the indicated polar residues (Fig. 2). Along the same lines, the ionizable amine may also serve to solubilize the tetrahydroisoquinoline ring. The 6,8 diol substituents of this tetrahydroisoquinoline are oriented toward the solvent interface.
Subsequent docking studies with other BoNT/A LC inhibitors from the NCI Diversity set indicated that these compounds may use binding modes similar to that of michellamine B. Fig. 3 shows a two-dimensional schematic that matches inhibitor substituents with proposed binding subsites for three inhibitors from the NCI Diversity set. Our analyses indicate that the most potent inhibitors engage in contacts with residues in both binding subsites 1 and 2, and in each case, either an oxygen or a nitrogen (in an aromatic heterocyclic ring) is positioned in close proximity to the catalytic zinc. For example, the 8-hydroxyquinoline of NSC 86372 (Fig. 3), when optimized in the substrate binding cleft, is positioned in such a way that zinc chelation is possible. Additionally, the methyl substituent of the compound's quinolinium substituent inserts into binding subsite 2 (similar to the methoxy moiety of michellamine B naphthalene B), while the ethoxy moiety of the quinolinium fills space located behind Pro 238 (similar to the methyl moiety of michellamine B naphthalene B).

In another example, the most favorable binding mode of NSC 357756 indicates that the dihydro-imidazolyl (attached to the benzofuran) inserts into binding subsite 1 (Fig. 3) (the same pocket that is occupied by the methoxy and methyl moieties of michellamine B naphthalene A (Fig. 2)). The oxygen of the benzofuran moiety is positioned in close proximity to the zinc ion, and the 3-amino substituent on the indole ring engages in a hydrogen bond with Glu 163. In binding subsite 2, a nitrogen in the dihydro-imidazolyl (which is attached to the 6 position of the indole) engages in a hydrogen bond

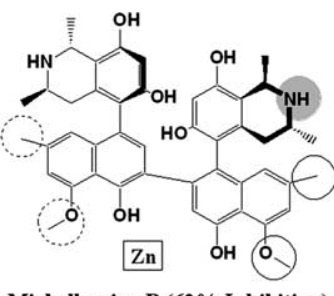

Michellamine B (62\% Inhibition)

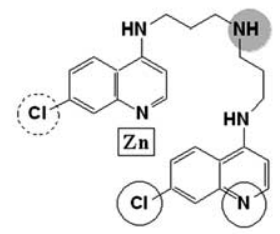

Q2-15 (60\% Inhibition)

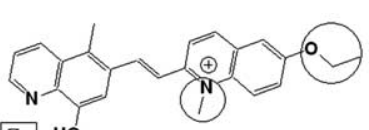

Zn HO NSC 86372 (51\% Inhibition)

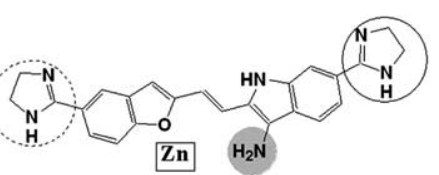

NSC 357756 (57\% inhibition)

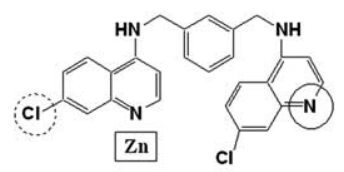

Q3-81 (32\% Inhibition)

\section{= Binding Subsite 1 Contact

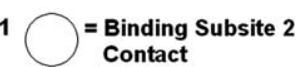 \\ Polar Residue Contact}

Fig. 3. A two-dimensional schematic relating compound substituents to predicted binding subsites in the BoNT/A LC substrate binding cleft. Dashed circles indicate substituents that engage in favorable contacts in binding subsite 1 , solid circles indicate substituents that engage in favorable contacts in binding subsite 2, and gray spheres indicate substituents that engage in favorable interactions with polar residues. 
with the side-chain guanidinium of Arg 230, while the methylenes of this ring pack behind the pyrrolidine ring of Pro 238.

\section{Structural evaluation and molecular docking of BQs and antimalarial drugs}

BQs possessing a flexible linker between the 7-chloro4-aminoquinoline moieties are more potent inhibitors than conformationally constrained derivatives (Table 2). In addition, it is also evident that an ionizable amine in the flexible linker increases potency (for example, Q2-61 versus Q1-19). Compound Q2-15 shows that a seven atom linker, with a central ionizable amine, is favored over shorter linkers that also contain an ionizable amine.

Conformational analyses of the most potent BQs show that these compounds favor a folded conformation, with the 7-chloro-4-aminoquinoline moieties positioned front to back and slightly offset relative to one another (Fig. 4A). Based on these observations, it appears that these congeners may all bind in the BoNT/A substrate binding cleft in a similar manner.

To gain a better understanding of how the folded conformations of these congeners might bind in the BoNT/A LC substrate cleft, Q2-15 was fit onto michellamine B (in its docked conformation). The superimposition shown in Fig. 4B indicates a good overlap between: (1) the 7-chloro-4-aminoquinoline components of Q2-15 and the naphthalene moieties of michellamine $\mathrm{B}$ and (2) the ionizable nitrogen of the Q2-15 flexible linker and the ionizable nitrogen of one of the michellamine $\mathrm{B}$ isoquinolines.

These observations led to docking studies to determine if the BQs might also bind to predicted subsites 1 and 2 in the BoNT/A LC substrate binding cleft. For the most potent compounds, the ring nitrogen of one of the 7-chloro-4-aminoquinoline moieties sits in close proximity to the catalytic zinc, while the 7-chloro substituent packs into binding subsite 1 . The ionizable amines in compounds with flexible linkers engage in potential ionic bonds with the polar side-chains of either Gln 161 or Glu 163. Another explanation for the importance of the ionizable amine may be that it is necessary for solubilizing the otherwise hydrophobic linkers found in this series of compounds.

Docking studies also indicate that the second 7chloro-4-aminoquinoline moiety (of the most potent derivatives) binds in subsite 2 such that the nitrogen of the heterocycle points toward the solvent and engages in a hydrogen bond with the side-chain guanidinium of Arg 230 (as was also observed for a nitrogen in one of the dihydro-imidazolyl rings of NSC 357756), and the 7chloro substituent points into the hydrophobic space behind His 226. For comparison, molecular docking of compounds with rigid linkers (for example, Q2-11 and Q2-97) indicates that steric constraints prevent these compounds from adopting binding modes in subsites 1 and 2 that are as favorable as those observed for congeners with flexible linkers. In general, these studies provide evidence that the BQs interact with the BoNT/A LC substrate binding cleft in a manner similar to inhibitors from the NCI Diversity set. Fig. 3 shows twodimensional schematics matching substituents from two of the most potent BQs (Q2-15 and Q3-81) with their corresponding binding subsites in the BoNT/A LC substrate binding cleft.

Of the five examined antimalarial drugs (Table 2), amodiaquine and quinacrine were the most potent $(30 \%$ inhibition of protease activity). However, the fact that these two drugs inhibit BoNT/A LC with approximately half the potency of Q2-15 provides further evidence that a second aromatic component, such as found in the BQs or michellamine $\mathrm{B}$, is necessary for enhanced potency, and supports our hypothesis that binding subsite 2 (in the enzyme's substrate binding cleft) is an important contact region for inhibitors. Furthermore, as quinacrine contains an acridine scaffold, these studies have revealed a new aromatic component that may be used to identify and/or synthesize new BoNT/A LC inhibitors.

The antimalarial agents that we found to inhibit BoNT/A LC protease activity have previously been shown to increase the time to BoNT/A holotoxin induced muscle paralysis $[17,18]$. In the earlier work, it was hypothesized that these compounds delayed paralysis by interfering with toxin translocation into the nerve cytoplasm and therefore they were not tested for specific inhibition of BoNT/A LC protease activity. The percent inhibition of BoNT/A LC that we observed for these drugs was not equivalent to the level of protection that they afforded against muscle paralysis in the previous studies $[17,18]$. For example, quinacrine, which we report to inhibit BoNT/A LC protease activity by $30 \%$ at $50 \mu \mathrm{M}(0.2 \mathrm{mM}$ substrate), was found, in an earlier study [17], to delay the time to $50 \%$ muscle paralysis by at least $30 \%$ (over control) at $3.3 \mu \mathrm{M}$ [17]. Thus, the results from the earlier studies, combined with the findings from our investigation, suggest that these compounds possess a dual mechanism of action-interfering with both BoNT/A entry into the cytoplasm and with the protease activity of the light chain. This dual mechanism of action is appealing from a drug design standpoint, as one molecule can be used to inhibit two separate functions of the same target. Indeed, if the BQs are found to also inhibit BoNT/A cytoplasm entry, then these studies will be the basis for the development of a new family of bifunctional inhibitors.

\section{A common pharmacophore for BoNT/A LC inhibitors}

Based on conformational sampling and molecular docking studies, a common pharmacophore for BoNT/ A LC inhibitors is proposed (Fig. 5A). An example of 

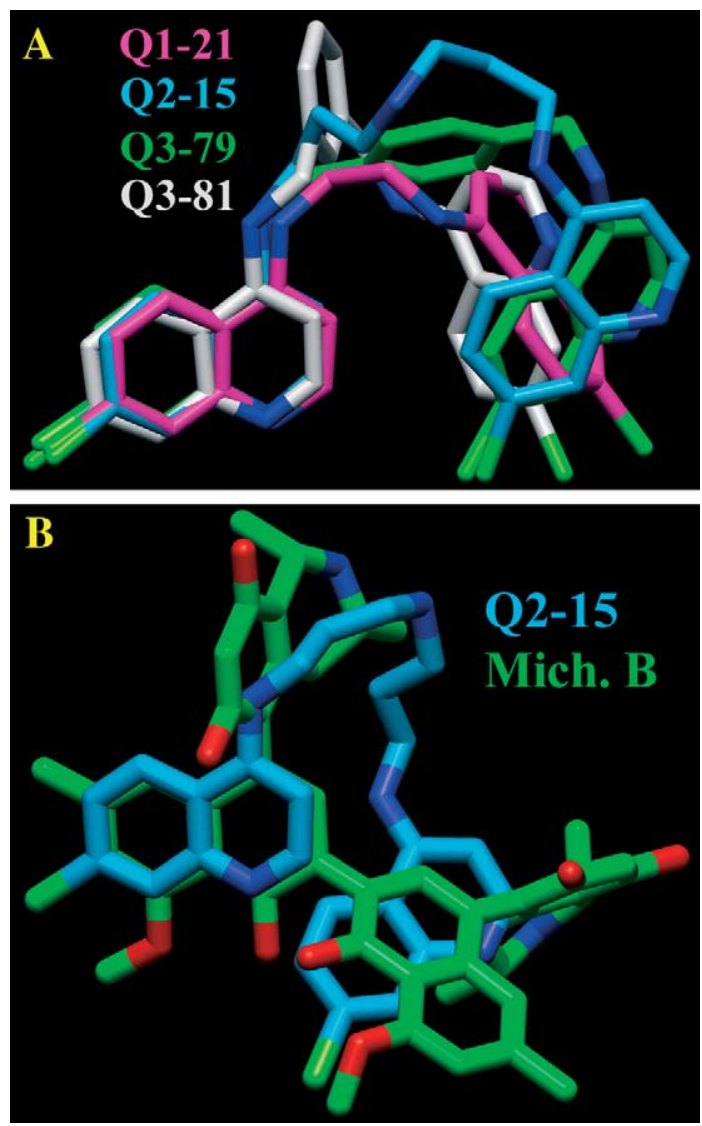

Fig. 4. (A) Overlay of folded conformers of BQs: Q1-21, Q2-15, Q3-79, and Q3-81. For all compounds, nitrogen atoms are dark blue and chlorine atoms are light green. The carbon atoms of Q1-21 are magenta, of Q2-15 are light blue, of Q3-79 are green, and of Q3-81 are white. A striking feature of the superimposed compounds is the close correspondence of their chloro substituents, which provides evidence that they may bind within the enzyme's substrate binding cleft in a similar manner. (B) Overlay of michellamine B and Q2-15. Carbons of michellamine B are green and carbon atoms of Q2-15 are light blue. The superimposed conformers show a good correspondence between: (1) the ionizable amine in the flexible linker of Q2-15 and the ionizable amine in one of the tetrahydroisoquinolines of michellamine B and (2) the chloro moieties of Q2-15 and the hydrophobic methoxy/methyl moieties of michellamine B.

how one of the inhibitors (Q2-15) fits the pharmacophore, using Catalyst 4.7 (Accelrys, San Diego, CA) as the query engine, is shown in Fig. 5B. Specifically, our studies show that the BoNT/A LC binding cleft can accommodate up to two biaryl/triaryl aromatic components, which are defined by planes $\mathrm{A}$ and $\mathrm{B}$ in the pharmacophore (Fig. 5A). These planes serve as scaffolds for positioning other components of the pharmacophore, provided that the distance between centroids of the two planes only varies from approximately $6.5-9.5 \AA$ (Fig. 5A). An additional requirement of plane $\mathrm{A}$ is the presence of a heteroatom (Fig. 5A) that may serve to either directly engage in an interaction with the catalytic zinc, or potentially replace the water molecule used by zinc during substrate lysis. Examples of structural
A
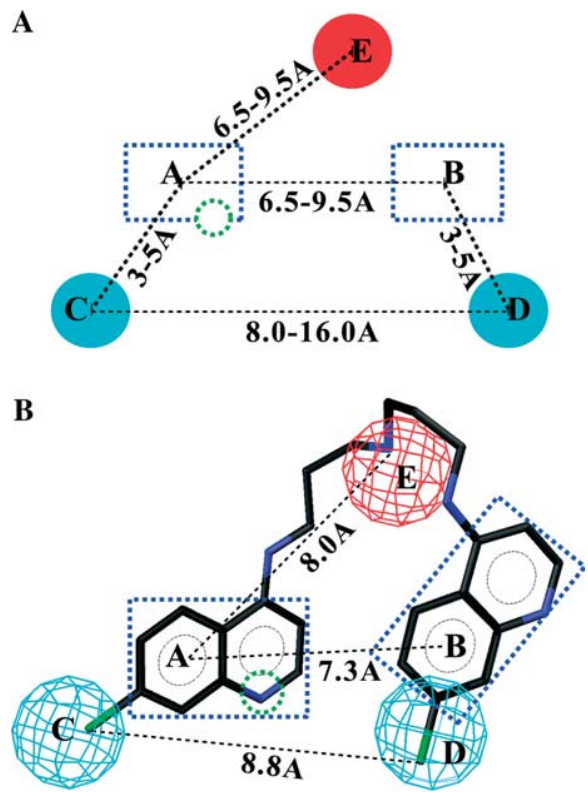

Fig. 5. (A) Common pharmacophore for BoNT/A LC inhibition. Planar components A and B are blue dashed rectangles. The dashed green circle in plane A represents a heteroatom. Hydrophobic components of the pharmacophore $(\mathrm{C}$ and $\mathrm{D})$ are shown with light blue spheres. The positive ionizable component of the pharmacophore $(\mathrm{E})$ is shown with a red sphere. (B) Q2-15 mapped onto the common pharmacophore (shown in (A)) in Catalyst 4.7 (Accelrys, San Diego, CA). Carbon atoms are black, nitrogen atoms are blue, and chlorine atoms are green. Planar components A and B are outlined with blue dashed rectangles. The heteroatom in plane $\mathrm{A}$ is indicated with a dashed green circle. Mapping of the hydrophobic components $(\mathrm{C}$ and $\mathrm{D})$ is indicated with light blue mesh spheres; mapping of the positive ionizable component $(\mathrm{E})$ is indicated with a red mesh sphere.

components of inhibitors that occupy planes $\mathrm{A}$ and $\mathrm{B}$ include: the two quinoline rings of $\mathrm{BQs}$ (a quinoline nitrogen would be the electron donor) (Table 2, Figs. 3, 4 , and 5B); the two naphthalene rings of michellamine B (a hydroxyl oxygen would be the electron donor) (Table 1, Figs. 2 and 3); and the indole and benzofuran rings of NSC 357756 (the benzofuran oxygen would be the electron donor) (Table 1 and Fig. 3).

Two hydrophobic moieties (C and D) are key components of the common pharmacophore (Fig. 5A). In relation to the BoNT/A LC substrate binding cleft, C and $\mathrm{D}$ correspond to binding subsites 1 and 2, and facilitate hydrophobic collapse between inhibitors and the enzyme. The $\mathrm{C} \rightarrow \mathrm{D}$ distance range of the common pharmacophore shown in Fig. 5A reflects the extent of the potential binding space that inhibitors may occupy in subsite 2 of the BoNT/A LC substrate binding cleft. Examples of $\mathrm{C}$ and $\mathrm{D}$ in inhibitors, as they map to the common pharmacophore, include: the two methoxy substituents of michellamine B (separated by a distance of $8.6 \AA$ ) (Table 1 and Figs. 2 and 3); the two chlorines of the BQs (separated by an average distance of $9.8 \AA$ for all examined BQs) (Table 2 and Figs. 3, 4, and 5B); 
and the two dihydro imidazolyl moieties of NSC 357756 (separated by a distance of $15.8 \AA$ ) (Table 1 and Fig. 3).

The final component (E) of the common pharmacophore is a positive ionizable substituent (Fig. 5A). While inhibitor docking studies have shown that an ionizable moiety may participate in electrostatic interactions with enzyme residues, the positive charge may also aid in solubilizing inhibitors bound within the BoNT/A LC substrate binding cleft; unlike a traditional binding pocket, the BoNT/A LC substrate binding cleft is large and almost completely solvent exposed. Hence, it is plausible that a hydrogen bonding network involving the positively charged moiety(ies) of the inhibitors and surrounding water molecules also serves to stabilize compound binding. Examples of inhibitor components that map to E include: the ionizable secondary nitrogen in one of the michellamine $\mathrm{B}$ tetrahydroisoquinolines (Table 1 and Figs. 2 and 3); the secondary nitrogens in the flexible linkers of BQs (Table 2 and Figs. 3, 4, and $5 \mathrm{~B})$; and the tertiary nitrogens in amodiaquine, chloroquine, and quinacrine (Table 2). Interestingly, the determined BoNT/A LC peptide cleavage site of the natural substrate, SNAP-25, is between a glutamine and an arginine [34]. Furthermore, the distance between the side-chain guanidinum of arginine (which is ionized at physiological $\mathrm{pH}$ ) and the backbone $\mathrm{C} \alpha$ of this residue corresponds to the distance between $\mathrm{A}$ and $\mathrm{E}$ in the common pharmacophore. Hence, it is possible that the ionizable substituent of the inhibitors may be mimicking contacts made by the side-chain guanidinium of the indicated Arg residue of SNAP-25.

\section{Summary}

These experiments have, for the first time, identified small organic (non-peptidic) lead therapeutics that inhibit BoNT/A LC protease activity in the low $\mu \mathrm{M}$ range. Based on the structures of these lead therapeutics, molecular modeling studies have been used to propose a common pharmacophore. This pharmacophore will serve as the basis for directing future database mining studies and synthetic organic chemistry projects to identify and develop BoNT/A LC inhibitors with enhanced potency.

\section{Acknowledgments}

The research described herein was sponsored by the US Army Medical Research and Material Command Research Plan \# 02-4-3U057 and IAA \# Y3-CM-100505 (MRMC and NCI). We acknowledge the National Cancer Institute for the allocation of computing time and staff support at the Advanced Biomedical Computing Center of the Frederick Cancer Research and Development Center. The content of this publication does not necessarily reflect the views or policies of the Department of Health and Human Services, nor does mention of trade names, commercial products, or organization imply endorsement by the US Government.

\section{References}

[1] J.J. Schmidt, R.G. Stafford, Fluorigenic substrates for the protease activities of botulinum neurotoxins, serotypes A, B, and F, Appl. Environ. Microbiol. 69 (2003) 297-303.

[2] K.R. Kessler, R. Benecke, Botulinum toxin: from poison to remedy, Neurotoxicology 18 (1997) 761-770.

[3] L.L. Simpson, Botulinum Neurotoxin and Tetanus Toxin, Academic Press, New York, 1989.

[4] R.L. Shapiro, C. Hatheway, D.L. Swerdlow, Botulism in the United States: a clinical and epidemiologic review, Ann. Intern. Med. 129 (1998) 221-228.

[5] D.R. Franz, P.B. Jahrling, A.M. Friedlander, D.J. McClain, D.L. Hoover, W.R. Byrne, J.A. Pavlin, G.W. Christopher, E.M. Eitzen Jr., Clinical recognition and management of patients exposed to biological warfare agents, JAMA 278 (1997) 399-411.

[6] S.S. Arnon, R. Schechtler, T.V. Inglesby, D.A. Henderson, J.G. Bartlett, M.S. Ascher, E. Eitzen, A.D. Fine, J. Hauer, M. Layton, S. Lillibridge, M.T. Osterholm, T. O’Toole, G. Parker, T.M. Perl, P.K. Russell, D.L. Swerdlow, K. Tonat, Botulinum toxin as a biological weapon: medical and public health management, JAMA 285 (2001) 1059-1070.

[7] M.C. Goodnough, G. Oyler, P.S. Fishman, E.A. Johnson, E.A. Neale, J.E. Keller, W.H. Tepp, M. Clark, S. Hartz, M. Adler, Development of a delivery vehicle for intracellular transport of botulinum neurotoxin antagonists, FEBS Lett. 513 (2002) 163168.

[8] D.B. Lacy, R.C. Stevens, Sequence homology and structural analysis of the clostridial neurotoxins, J. Mol. Biol. 291 (1999) 1091-1104.

[9] K. Turton, J.A. Chaddock, K.R. Acharya, Botulinum and tetanus neurotoxins: structure, function and therapeutic utility, Trends Biochem. Sci. 27 (2002) 552-558.

[10] B.R. Singh, Intimate details of the most poisonous poison, Nat. Struct. Biol. 7 (2000) 617-619.

[11] T. Binz, J. Blasi, S. Yamasaki, A. Baumeister, E. Link, T.C. Sudhof, R. Jahn, H. Niemann, Proteolysis of SNAP-25 by types E and A botulinal neurotoxins, J. Biol. Chem. 269 (1994) 1617-1620.

[12] G. Schiavo, F. Benfenati, B. Poulain, O. Rossetto, P. Polverion de Laureto, B.R. DasGupta, C. Montecucco, Tetanus and botulinum-B neurotoxins block neurotransmitter release by proteolytic cleavage of synaptobrevin, Nature 359 (1992) 832-835.

[13] G. Schiavo, O. Rossetto, S. Catsicas, P. Polveriono de Laureto, B.R. DasGupta, F. Benfenati, C. Montecucco, Identification of the nerve terminal targets of botulinum neurotoxin serotypes A, D, and E, J. Biol. Chem. 268 (1993) 23784-23787.

[14] G. Schiavo, C.C. Shone, O. Rossetto, F.C. Alexander, C. Montecucco, Botulinum neurotoxin serotype $\mathrm{F}$ is a zinc endopeptidase specific for VAMP/synaptobrevin, J. Biol. Chem. 268 (1993) 11516-11519.

[15] G. Schiavo, C. Malizio, W.S. Trimble, P. Polveriono de Laureto, G. Milan, H. Sugiyama, E.A. Johnson, C. Montecucco, Botulinum G neurotoxin cleaves VAMP/synaptobrevin at a single AlaAla peptide bond, J. Biol. Chem. 269 (1994) 20213-20216.

[16] J. Blasi, E.R. Chapman, S. Yamasaki, T. Binz, H. Niemann, R. Jahn, Botulinum neurotoxin $\mathrm{C} 1$ blocks neurotransmitter release by means of cleaving HPC-1/syntaxin, EMBO J. 12 (1993) 48214828.

[17] R.E. Sheridan, S.S. Deshpande, J.D. Nicholson, M. Adler, Structural features of aminoquinolines necessary for antagonist activity against botulinum neurotoxin, Toxicon 35 (1997) 14391451. 
[18] S.S. Deshpande, R.E. Sheridan, M. Adler, Efficacy of certain quinolines as pharmacological antagonists in botulinum neurotoxin poisoning, Toxicon 35 (1997) 433-445.

[19] J. Hayden, J. Pires, S. Roy, M. Hamilton, G.J. Moore, Discovery and design of novel inhibitors of botulinus neurotoxin A: targeted 'hinge' peptide libraries, J. Appl. Toxicol. 23 (2003) 1-7.

[20] J.J. Schmidt, R.G. Stafford, K.A. Bostian, Type A botulinum neurotoxin proteolytic activity: development of competitive inhibitors and implications for substrate specificity at the S1' binding site, FEBS Lett. 435 (1998) 61-64.

[21] J.J. Schmidt, R.G. Stafford, A high-affinity competitive inhibitor of type A botulinum neurotoxin protease activity, FEBS Lett. 532 (2002) 423-426.

[22] J.J. Schmidt, R.G. Stafford, C.B. Millard, High-throughput assays for botulinum neurotoxin proteolytic activity: serotypes A, B, D, and F, Anal. Biochem. 296 (2001) 130-137.

[23] J.L. Vennerstrom, W.Y. Ellis, A.L. Ager Jr., S.L. Andersen, L. Gerena, W.K. Milhous, Bisquinolines. 1. N,N-bis(7-chloroquinolin-4-yl)alkanediamines with potential against chloroquine-resistant malaria, J. Med. Chem. 35 (1992) 2129-2137.

[24] J.L. Vennerstrom, A.L. Ager Jr., A. Dorn, S.L. Andersen, L. Gerena, R.G. Ridley, W.K. Milhous, Bisquinolines. 2. Antimalarial $N, N$-bis(7-chloroquinolin-4-yl)heteroalkanediamines, $\mathrm{J}$. Med. Chem. 41 (1998) 4360-4364.

[25] S.A. Ahmed, L.A. Smith, Light chain of botulinum A neurotoxin expressed as an inclusion body from a synthetic gene is catalytically and functionally active, J. Prot. Chem. 19 (2000) 475-487.

[26] J.J. Schmidt, K.A. Bostian, Endoproteinase activity of type A botulinum neurotoxin: substrate requirements and activation by serum albumin, J. Prot. Chem. 16 (1997) 19-26.

[27] D.B. Lacy, W. Tepp, A.C. Cohen, B.R. DasGupta, R.C. Stevens, Crystal structure of botulinum neurotoxin type A and implications for toxicity, Nat. Struct. Biol. 5 (1998) 898-902.
[28] P. Giannakakou, R. Gussio, E. Nogales, K.H. Downing, D. Zaharevitz, B. Bollbuck, G. Poy, D. Sackett, K.C. Nicolaou, T. Fojo, A common pharmacophore for epothilone and taxanes: molecular basis for drug resistance conferred by tubulin mutations in human cancer cells, PNAS 97 (2000) 2904-2909.

[29] M.G.A. Korn, A.C. Ferreira, L.S.G. Teixeira, A.C.S. Costa, Spectrophotometric determination of zinc using 7-(4-nitrophenylazo)-8-hydroxyquinoline-5-sulfonic acid, J. Braz. Chem. Soc. 10 (1999) 46-50.

[30] Y.F. Hallock, K.P. Manfredi, J.-R. Dai, J.H. Cardellina II, R.J. Gulakowski, J.B. McMahon, M. Schaffer, M. Stahl, K.-P. Gulden, G. Bringmann, G. Francois, M.R. Boyd, Michellamines D-F, new HIV-inhibitory dimeric naphthylisoquinoline alkaloids, and korupensamine E, a new antimalarial monomer, from Ancistrocladus korupensis, J. Nat. Prod. 60 (1997) 677-683.

[31] K.P. Manfredi, J.W. Blunt, J.H. Cardellina II, J.B. McMahon, L.L. Pannell, G.M. Cragg, M.R. Boyd, Novel alkaloids from the tropical plant Ancistrocladus abbreviatus inhibit cell killing by HIV-1 and HIV-2, J. Med. Chem. 34 (1991) 3402-3405.

[32] M.R. Boyd, Y.F. Hallock, J.H. Cardellina II, K.P. Manfredi, J.W. Blunt, J.B. McMahon, R.W. Buckheit Jr., G. Bringmann, M. Schaffer, G.M. Cragg, D.W. Thomas, J.G. Jato, Anti-HIV michellamines from Ancistrocladus korupensis, J. Med. Chem. 37 (1994) 1740-1745.

[33] G. Bringmann, R. Zagst, M. Schaffer, Y.F. Hallock, J.H. Cardellina II, M.R. Boyd, The absolute configuration of michellamine B, a dimeric, anti-HIV-active naphthylisoquinoline alkaloid, Angew. Chem. Int. Ed. Engl. 32 (1993) 1190-1191.

[34] V.V. Vaidyanathan, K.-I. Yoshino, M. Jahnz, C. Dorries, S. Bade, S. Nauenburg, H. Niemann, T. Binz, Proteolysis of SNAP-25 isoforms by botulinum neurotoxin types $\mathrm{A}, \mathrm{C}$, and $\mathrm{E}$ : domains and amino acid residues controlling the formation of enzyme-substrate complexes and cleavage, J. Neurochem. 72 (1999) 327-337. 